\title{
Modeling of an EMP Simulator Using a 3-D FDTD Code
}

\author{
Shahid Ahmed, Deepti Sharma, and Shashank Chaturvedi
}

\begin{abstract}
We have performed self-consistent, three-dimensional (3-D), time-domain calculations for a bounded-wave electromagnetic pulse simulator. The simulator consists of a constantimpedance transverse electromagnetic structure driven by a charged capacitor, discharging through a fast closing switch. These simulations yield the detailed 3-D electromagnetic field structure in the vicinity of the simulator.

The prepulse seen in these simulations can be explained quantitatively in terms of capacitive coupling across the switch and the known charging waveform across the capacitor.

Placement of a test object within the simulator significantly modifies the electric fields within the test volume, in terms of field strength as well as the frequency spectrum. This means that, for a given simulator, larger objects would be subjected to somewhat lower frequencies. The E-field waveform experienced by a small test object is reasonably close to that for free-space illumination, but the mismatch increases with object size.

The use of a resistive sheet as a matching termination significantly reduces radiation leakage as compared to two parallel resistive rods. For a given termination, larger test objects marginally reduce leakage. A physical interpretation of these conclusions is also included.

This work is a first step toward full-fledged optimization of such simulators using 3-D modeling.
\end{abstract}

Index Terms-Bounded-wave, electromagnetic interference (EMI), electromagnetic pulse (EMP), finite-difference time-domain (FDTD), prepulse, transverse electromagnetic (TEM).

\section{INTRODUCTION}

$\mathbf{E}$ LECTROMAGNETIC pulse (EMP) simulators are widely used for testing equipment susceptibility to EMP. A bounded-wave EMP simulator, in its simplest form, consists of two electrically conducting triangular plates separated by a parallel plate region [1]. A capacitor bank, charged to high voltage, is discharged through a fast closing switch into the front triangular plate. The current flows through the structure to the rear triangular plate, then through a matching termination, and back through a conducting ground plane. The front plate, which displays a near-constant impedance over a wide frequency range, plays a significant role in determining the EMP waveform, while the middle and rear plates serve to guide the signal [1]. The object to be tested is mounted in the bounded volume of the parallel-plate region. The discharging capacitor produces an intense, rapidly varying electromagnetic field, covering a wide frequency range, in the vicinity of the test object [2].

Manuscript received September 26, 2001; revised October 28, 2002.

The authors are with the Institute for Plasma Research, Gandhinagar 382428, India (e-mail: shahid@ipr.res.in).

Digital Object Identifier 10.1109/TPS.2003.810190

\section{A. Need for FDTD Analysis}

The high cost of setting up EMP simulators means that fabrication must be preceded by numerical optimization. The general behavior of a simulator is complex due the existence of a wide-band pulse and complex geometry of the simulator and test object. It can be further complicated by the presence of different materials, both dielectrics and conductors, in the test object. The transverse electromagnetic (TEM) structure-capacitorswitch-test object combination is a complex three-dimensional (3-D) object. The input impedance $Z(\omega)$ of the TEM structure is a function of frequency. $\vec{E}(\omega)$ inside the structure depends upon the input current waveform $I(t)$, which, in turn, depends upon $Z(\omega)$. This means that the electric field distribution within the structure, and $I(t)$, must be determined self-consistently. Hence, there is a need for a self-consistent 3-D analysis.

An important output required of such an analysis is the electromagnetic field variation, $\vec{E}(\vec{r}, t)$ and $\vec{B}(\vec{r}, t)$, in the vicinity of the test object. Now, the object could itself be complex, consisting of various materials, having internal cavities, small apertures, and so on. Placement of the object within the test volume would, therefore, modify the fields to which it is subjected. The fields are further affected by scattering of waves off the object as well as the simulator structure. This means that details of the simulator, such as launcher angles, finite plate widths, earth conductivity, etc., would also play a role [3].

Several time- and frequency-domain models have been reported for the analysis of EMP simulators. These analyses are based on several simplifying assumptions. For example, the conducting plates of a simulator have been approximated by wire grids or meshes. The current induced on the wires is solved in the time- or frequency-domain using a space-time-domain technique [4] or the method-of-moments (MOM) [5]. The transient electromagnetic field distribution inside a simulator has been studied through a space-time-domain technique [4]. MOM has been used to analyze a high-frequency band of the EMP spectrum [5]. These methods are not suited for detailed analysis of simulators with test objects, for two reasons. First, these techniques tend to ignore skin effects. This may be acceptable for the simulator plates, but not for conducting test objects, particularly over the low-frequency portion of EMP. Secondly, MOM is not suited for handling objects with small apertures and internal cavities.

The finite-difference time-domain (FDTD) method is a powerful tool for analyzing problems involving 3-D objects with complex geometries and multiple materials [6]. Hence, it is ideally suited for analysis of EMP simulators, and is used in the present work. 


\section{B. Choice of Method for Self-Consistent Analysis}

In certain studies of EMP simulators, the excitation is assumed to follow a known form, e.g., a double exponential. Other workers have reported 3-D FDTD studies of horn antennas driven by specified excitation pulses [7], [8]. The excitation pulse is usually taken either as an idealized form like a Gaussian [7], [8], or an experimentally-measured waveform [8]. To our knowledge, none of these studies have self-consistently evolved the pulser (e.g., capacitor bank) voltage along with the electromagnetic fields in the structure.

A self-consistent analysis could be done in two ways. The first would be to model the simulator structure through the 3-D FDTD equations [6], and treat the capacitor and switch as lumped elements whose evolution is represented by ordinary differential equations. The two sets of equations would be coupled at the feed point of the TEM structure by matching the voltage and current. This would require an iterative solution at each FDTD time-step, which is computationally expensive. The second method would be to set up the capacitor and switch, along with the simulator structure, in the FDTD grid itself. This would, naturally, require idealizations to be made in the geometry of the capacitor and switch. However, assuming that the geometry is reasonably well represented, this method offers the advantage of taking into account distributed parameter effects within the capacitor and switch, which could be significant for short time-scale phenomena. The addition of the switch and capacitor marginally increases the size of the FDTD domain, with a resulting increase in the computational effort per time-step. However, this is more than compensated by avoiding the iterations which are inherent in the first method. We have, therefore, opted for the second approach.

\section{Scope of This Work}

We have divided the study of a bounded-wave EMP simulator into two parts. In Part I, consisting of Sections II-V, we consider the performance of the capacitor-switch-TEM structure, with a resistive termination placed at the end of the tapered section. This means that the test volume and test object are not included, i.e., we only examine the formation and propagation of an EMP. In Part II, consisting of Sections VI and VII, we model the structure shown in Fig. 1, where the termination is placed at the end of a parallel-plate extension, and a simple test object is placed within that extension. Here, we examine the interaction of the EMP with a test object, and provide a physical interpretation of some of the results.

Section II describes the simulator examined in this study, as well as details of the computational model. In Section III, we present results of electromagnetic field structure within the bounded space. Section IV examines radiation leakage from the system, an important issue from the point of view of electromagnetic compatibility. The prepulse seen before switching is discussed in Section V. Simulator performance in the presence of the test object is examined in Section VI. Section VII presents a physical interpretation of some of the foregoing results. Major limitations of the study are summarized in Section VIII and the conclusions in Section IX.

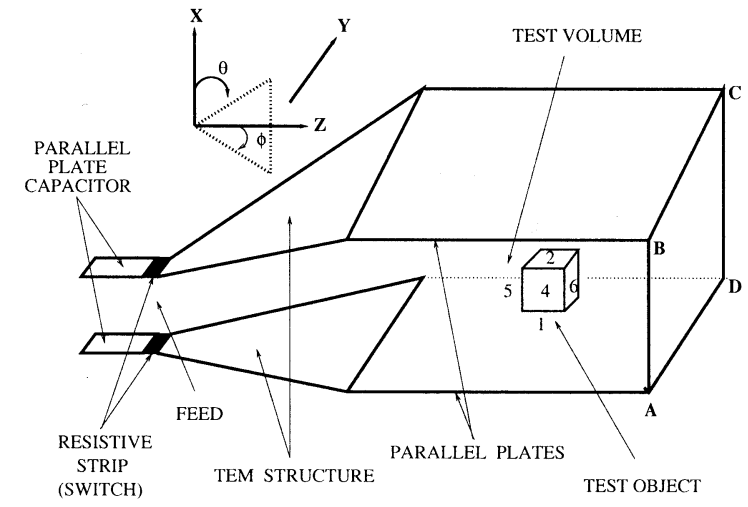

ABCD : $90 \Omega$ SHEET TERMINATION

Fig. 1. Schematic of EMP simulator with parallel-plate test volume and resistive sheet termination.

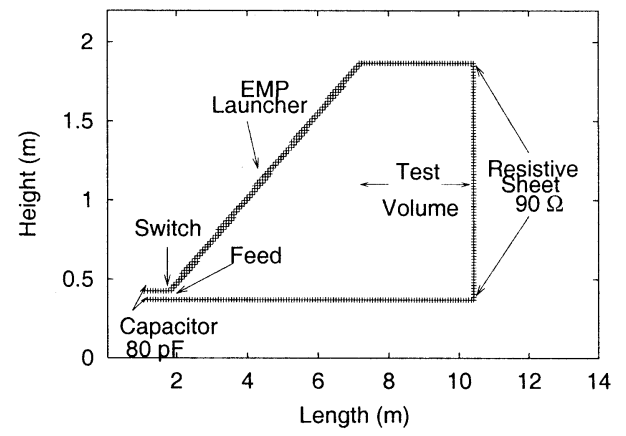

Fig. 2. Cross-sectional elevation of FDTD mesh.

\section{DetaILS OF MODEL}

Fig. 1 shows a schematic of the system being modeled. For purposes of illustration, the parameters of the pulser and the TEM structure are chosen to be similar to those of the EMP simulator described in [2]. The TEM structure of [2] has a characteristic impedance of $90 \Omega$ and a matching resistive termination at the aperture. It is driven by an $82-\mathrm{pF}$ peaking capacitor charged to $1 \mathrm{MV}$. Only limited data is available about that simulator. To facilitate the analysis, and in some cases due to lack of data, we have made certain simplifying assumptions which are described in Section VIII. In particular, the test volume of the simulator is not shown in [2], hence, we have assumed a small test volume as shown in Fig. 1. The objective of this study is to illustrate the utility of 3-D FDTD calculations for such simulators, rather than an exact match with experiment. The method can readily be adapted to handle more realistic designs.

We have used a 3-D FDTD code [6] for this work. Fig. 2 shows a cross-sectional elevation of the FDTD mesh. The peaking capacitor, which consists of a set of water capacitors in [2], is idealized as a single parallel-plate air-gap capacitor in our model. Both plates of the capacitor and the simulator are assumed to be made of perfect electrical conductor (PEC). The peaking capacitor is charged to $\sim 1 \mathrm{MV}$. Our simulation starts with an uncharged capacitor, and charging is accomplished by the application of a voltage waveform following a half-Gaussian in time-details are given in Section V.

The computational mesh changes according to the needs of each problem. A typical mesh consists of $122 \times 156 \times 365$ 
cells in the $x, y$, and $z$ directions, with uniform cell sizes $\Delta x$, $\Delta y$, and $\Delta z$ of $1.85 \mathrm{~cm}$. This corresponds to a time step of $35.6 \mathrm{ps}$ according to the Courant criterion [6]. The active region, consisting of the capacitor, switch and TEM structure, is placed within this domain with a uniform spacing of at least 20 cells to the domain boundaries.

A simplified model has been used for the closing switch, as shown in Figs. 1 and 2. This consists of two resistive strips, each with a length of one computational cell, separating the upper and lower capacitor plates from the corresponding plates of the TEM structure. The strip material has a time-dependent resistivity, which is initially kept infinite during the charging of the capacitor. This means that no charge can move to the simulator structure while the capacitor is being charged. However, a prepulse is still possible due to capacitive coupling. This is examined in Section V.

The actual temporal variation of switch resistivity would depend upon the physics of the breakdown process. Since detailed modeling of the switch is beyond the scope of this work, we must choose an approximate waveform that is physically justifiable. It is well known that many fast closing switches, involving breakdown of a gas or liquid, exhibit a statistical time-lag [9]. Starting from the time at which a strong electric field is applied, there is a slow buildup of the avalanche breakdown process, during which the effective resistance of the switch remains high. Following this time lag, the avalanche builds up rapidly, leading to rapid voltage collapse across the electrodes.

A waveform that yields this kind of behavior, from the fully-open to the fully-closed state, is given by the "half-Gaussian"

$$
\sigma=\sigma_{c} \exp \left[-\alpha_{s}\left(t-\tau_{s}-\tau_{0}\right)^{2}\right] \quad \tau_{0} \leq t \leq \tau_{s}+\tau_{0}
$$

where $\tau_{s}$ is a characteristic time for closure, $\tau_{0}$ is the time when switching starts, $\alpha_{s}=\left(4 / \tau_{s}\right)^{2}$, and $\sigma_{c}$ is the switch material conductivity in the fully-closed state. $\tau_{s}$ is chosen so as to avoid the generation of frequencies higher than those that can be handled by our FDTD mesh [6]. $\sigma_{c}$ is chosen such that, in the fully closed state, the switch resistance becomes very small in comparison with the characteristic impedance of the TEM structure.

We see that the conductance remains small until " $t$ " comes fairly close to $t=\tau_{s}+\tau_{0}$, followed by a small interval exhibiting rapid growth in $\sigma$. Hence, the "time lag" is reproduced to some extent.

Another advantage with the half-Gaussian waveform is that its first derivative falls to zero in the fully closed state, which should reduce the spurious generation of high frequencies.

Let us now consider another property of this waveform. We see that, at the start of switching, i.e., $t=\tau_{0}$ in (1), there is a discontinuity in the conductance, which is taken to be zero during capacitor charging. However, the magnitude of the discontinuity is small, being $\sim 7$ orders of magnitude smaller than the peak conductance in the fully closed state. This step-change would, naturally, excite all frequencies. Now, the FDTD mesh can accurately handle frequencies up to some upper bound determined by mesh sizes. However, since the step change has a small magnitude, it is numerically acceptable [6].

A question that naturally arises is whether some other waveform might not be superior to the half-Gaussian in some or all of these areas. From the point of view of the temporal derivatives of $\sigma$ at $t=\tau_{s}+\tau_{0}$, an error function waveform would be better, since both the first- and second-derivatives would become zero in the fully-closed state. The error function, however, suffers from two major disadvantages. The first is that it does not exhibit the "time delay" discussed previously. Indeed, it would yield a $d \sigma / d t$ waveform that has its maximum at $t=\tau_{0}$ and steadily decreases as we move toward the fully-closed state. Secondly, it has a problem around the time switching starts, as explained in the following.

The characteristic frequency $f_{c}$ for variation of $\sigma$ can be estimated from

$$
f_{c}=\frac{1}{\sigma} \frac{d \sigma}{d t}
$$

where $\sigma=\sigma_{0} \times \operatorname{erf}(x)$ and $x=\left(t-\tau_{0}\right) / \tau_{s}$. Substituting for $\sigma$, we get

$$
f_{c}=\frac{2}{\tau_{s} \sqrt{\pi}} \cdot \frac{e^{-x^{2}}}{\operatorname{erf}(x)} .
$$

This frequency is a function of time. When switching starts at $t=\tau_{0}$, we have $\sigma=0$, hence, this would yield an infinitely high frequency.

To get around this problem, we could start at a finite value of $\sigma$, as in the half-Gaussian model, by redefining $x=(t-$ $\left.\tau_{0}+\delta \tau_{s}\right) / \tau_{s}$. This is where a major difference between the half-Gaussian and error function waveforms becomes apparent. In the Gaussian form, at small $\sigma / \sigma_{0}$ values, both the function and its derivative are small, so that the characteristic frequency remains reasonable. With $\operatorname{erf}(x)$, however, the derivative is initially large and progressively decreases in time. Hence, $f_{c}$ can become very large when switching starts. For example, starting with $\sigma=10^{-7} \sigma_{0}$, i.e., $\delta=8.863 \times 10^{-8}$, and $\tau_{s}=19.7 \mathrm{~ns}$, we get $f_{c}=5.7 \times 10^{14} \mathrm{~Hz}$, which is far too high.

We could further increase the initial value of $\sigma / \sigma_{0}$ to overcome this problem. Let us say we want to restrict $f_{c}$ to below $400 \mathrm{MHz}$, the limit of the computational mesh in one problem. This requires $\sigma / \sigma_{0}=0.15$ at $t=\tau_{0}$. This, however, raises its own problems - the initial jump in $\sigma$ is now considerable, and the resulting generation of high frequencies introduces substantial errors into the FDTD calculation.

Fig. 3 shows the effect of choosing $\sigma / \sigma_{0}=10^{-7}$ and 0.15 , respectively, on the observed E-field waveform inside the TEM structure. There is significant high-frequency noise, which is not observed with the half-Gaussian waveform. We believe that this noise is due to the high frequencies generated by the error function.

We conclude, therefore, that a half-Gaussian waveform is better suited to model switching, despite its limitations as regards continuity of the second derivative at the peak.

These simulations yield the temporal behavior of the input current to the TEM structure and the 3-D distribution of electromagnetic field within the structure.

\section{ELECTRIC FIELD INSIDE TEM STRUCTURE}

We now present results for the case of a charged capacitor discharging through the TEM structure terminated by a perfectly matched sheet $(90 \Omega)$. In these simulations, the main component 


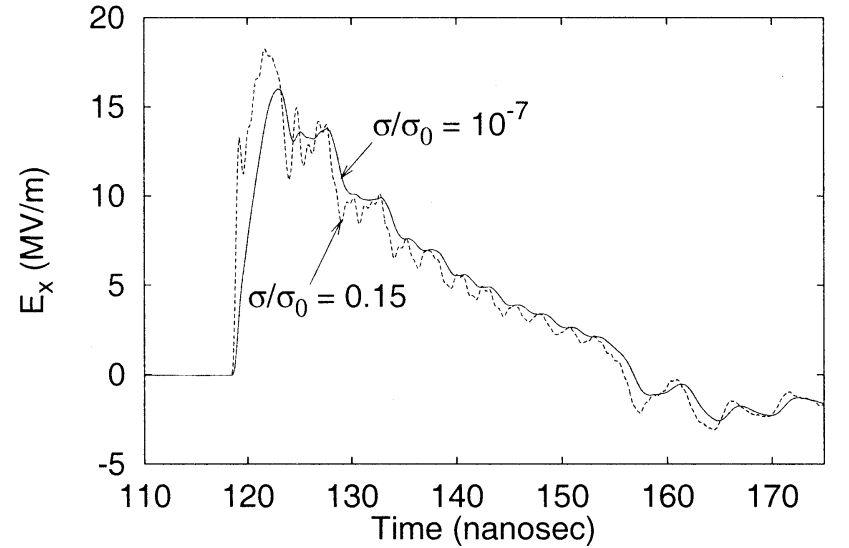

Fig. 3. Vertical component of electric field at TEM structure feed for different values of $\sigma\left(t=\tau_{0}\right) / \sigma_{0}$, using the error function waveform for switching.

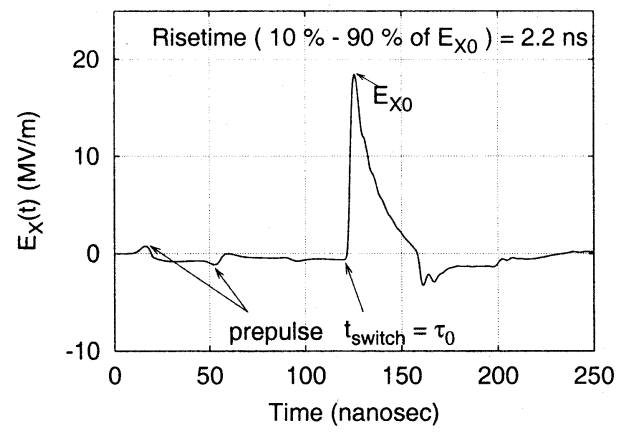

Fig. 4. Vertical component of electric field at TEM structure feed.

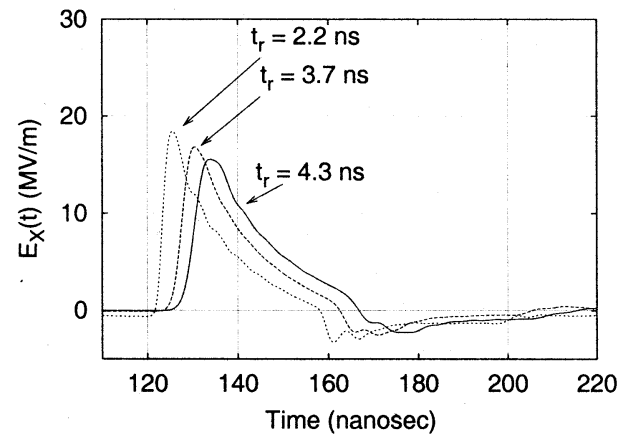

Fig. 5. Electric field waveform at feed point for different switching times. Case with matching $90-\Omega$ resistive termination. The prepulse is omitted for clarity.

of the electric field is taken as $E_{x}$, where " $x$ " is the height, as shown in Fig. 2. Fig. 4 illustrates the general nature of $E_{x}(t)$ at the feed point, showing both the prepulse (before switching) and the main pulse (after switching). An arrow marks the time $\tau_{0}$ when switching starts. A detailed discussion of the prepulse is presented in Section V. In the rest of this section, we focus on the main pulse.

\section{A. Effect of Switch Closure Time}

For the case of a TEM structure with a $90-\Omega$ resistive termination, we have studied the effect of varying the switching time. Fig. 5 shows the main pulse at the feed point for three different values of $\tau_{s}$, viz., 19.7, 14.8, and 7.4 ns. Runs with faster switching times require a finer FDTD mesh, so the capacitance $C_{0}$ and charging voltage $V_{0}$ of the parallel-plate capac-

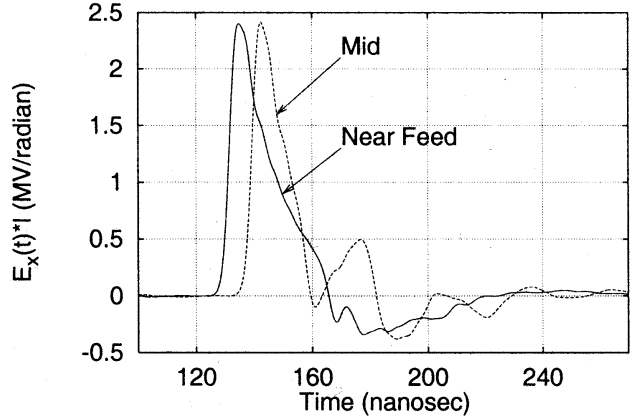

Fig. 6. Vertical electric field $E_{x} * l$ at two locations along boresight. Case with matched termination and $\tau_{s}=19.7 \mathrm{~ns}$.

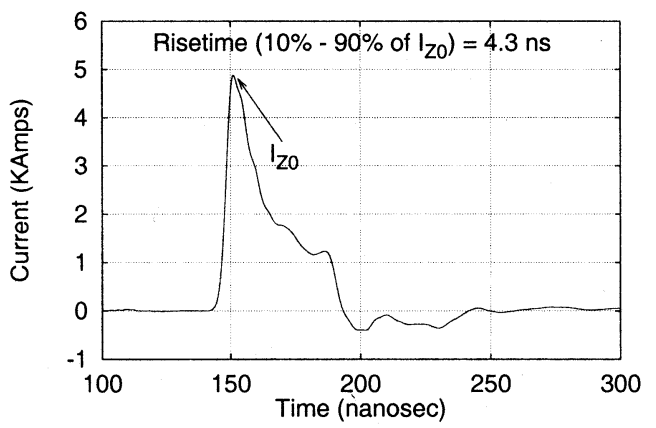

Fig. 7. $Z$-directed current flowing through lower plate near the aperture. Case with matched termination and $\tau_{s}=19.7$ ns.

itor are slightly different in the three cases, viz. $V_{0}=917,911$, and $906 \mathrm{kV}$ and the corresponding $C_{0}=85,82$, and $80 \mathrm{pF}$, respectively. We observe a $10 \%-90 \%$ risetime for $E_{x}(t)$, i.e., $\tau_{E}=4.3,3.7$, and $2.2 \mathrm{~ns}$ in the three cases. These correspond to $0.22 \tau_{s}, 0.25 \tau_{s}$, and $0.30 \tau_{s}$, respectively.

This relation between $\tau_{s}$ and $\tau_{E}$ can be understood as follows. The switch conductivity waveform of (1) implies that $\sigma$ rises from $0.2 \sigma_{c}$ to $\sigma_{c}$ in $\sim 0.32 \tau_{s}$. It is reasonable to assume that the flow of charge from the capacitor to the TEM structure only becomes significant when $\sigma$ is high enough, say $\sigma>0.2 \sigma_{c}$. It is this flow of charge that builds up the electric field inside the TEM structure. Hence, a risetime in the range $0.2-0.3 \tau_{s}$ for $E_{x}(t)$ in the TEM structure is understandable. It is not presently clear why the ratio $\tau_{E} / \tau_{s}$ should increase as we reduce the risetime. It is likely that the inductance of the connection region between the capacitor and TEM structure, including the switch, starts playing a role for very fast risetimes.

Fig. 6 shows $E_{x}(t)^{*} L$ in the main pulse at two locations along the boresight of the TEM structure, viz., near the feed point and midway between the feed and aperture, for the case with $\tau_{s}=19.7 \mathrm{~ns}$. Here, " $L$ " is the distance of the observation point from the apex of the TEM structure. We note that the risetime and pulsewidth are about the same at both points, as expected.

For the case with $\tau_{s}=19.7 \mathrm{~ns}$, Fig. 7 shows the temporal waveform of the $z$-directed current flowing through the lower plate near the aperture. This current has been obtained through the path-integral $\int \vec{H} \cdot d \vec{l}$. The observed time-delay of $16.5 \mathrm{~ns}$ between the peak current in Fig. 7 and the peak $E_{x}^{*} L$ near the feed in Fig. 6 is consistent with the transit time between these points. 


\section{B. Choice of Distance to Domain Boundary}

A second-order outer radiation boundary condition (ORBC) has been used in the simulations [6]. ORBC is applicable only if the distance between the simulator and the domain boundaries is at least of the order of a wavelength [6]. This condition becomes difficult to satisfy when the simulation involves an ultra-wideband spectrum. On the one hand, the total domain size would be governed by the lowest frequency. At the same time, the mesh size must be dictated by the highest frequency of interest. This combination would require unacceptably large numbers of computational cells. It is clearly impossible to satisfy this condition for very low frequencies, e.g., for the long-time response. It has been reported in [7] that good agreement is obtained between simulation and experiments when the above condition is satisfied for the highest frequency of interest.

We have performed Fourier analysis of $E_{x}(t)$ at the feed point to identify the highest frequency of interest $f_{\max }$ for different switching times. This is defined as the frequency where $\left|E_{x}(f)\right|$ falls to 0.001 of its peak value. The distance to the domain boundary has then been chosen appropriately.

\section{EMI DUE TO SIMULATOR}

The minimization of electromagnetic interference (EMI) outside a bounded-wave simulator requires the reduction of radiated power. It is well known that while the lower frequencies are absorbed by a matched termination, a good part of the energy contained in the high frequency regime is lost by radiation. Hence, it is impossible to stop the radiation completely if one uses a TEM structure as an EMP simulator.

In experimental systems, several parallel chains of resistors are often used as the termination. It is of interest to examine the two limiting cases of this arrangement. The first limit is a resistive sheet, which would arise if the spacing between adjacent chains were made significantly smaller than the wavelengths of interest. The second limit consists of two resistive rods connecting corresponding corners of the upper and lower plates.

One way of estimating leakage would be to calculate the far-field from the structure and integrate over a bounding surface. We have opted for a simpler method, as follows. The net power outflow from the FDTD domain $P_{\text {net }}$ is calculated by taking the surface integral of the Poynting flux, $\vec{E} \times \vec{B}$, over the boundaries of the FDTD domain. It must be noted that the instantaneous power outflow consists of both radiative and reactive terms. The reactive power refers to changes in energy stored in the near field, and can be either positive or negative. The radiative power refers to power entering the far field. Hence, a negative value of $P_{\text {net }}$ means that the inward-directed reactive component exceeds the radiated power at that moment.

Fig. 8 shows the temporal evolution of $P_{\text {net }}$ for two cases, using matching sheet and rod terminations respectively. The matched rod termination consists of two parallel rods, each of resistance $180 \Omega$, connecting corresponding corners of the upper and lower plates. In both cases, we have $\tau_{s}=19.7 \mathrm{~ns}$. The simulations are run until $P_{\text {net }}$ falls to very low levels, which happens by around $0.24 \mu \mathrm{s}$ in both cases. The area under the curve, which yields the total outflow energy, is $6.07 \mathrm{~J}$ and $7.94 \mathrm{~J}$ for sheet and

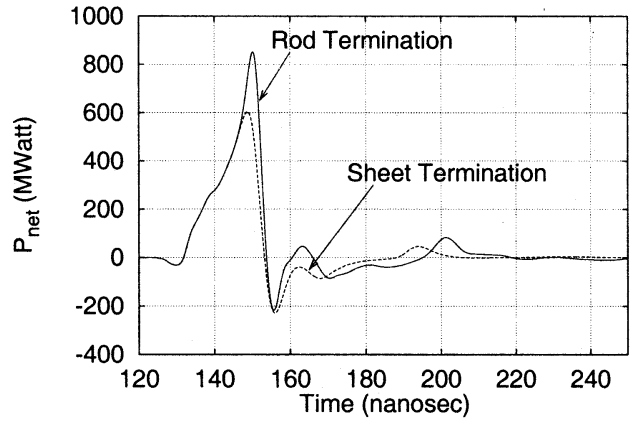

Fig. 8. Net outward power flow from FDTD domain, with matched resistive termination. Curves shown for both sheet and rod geometries.
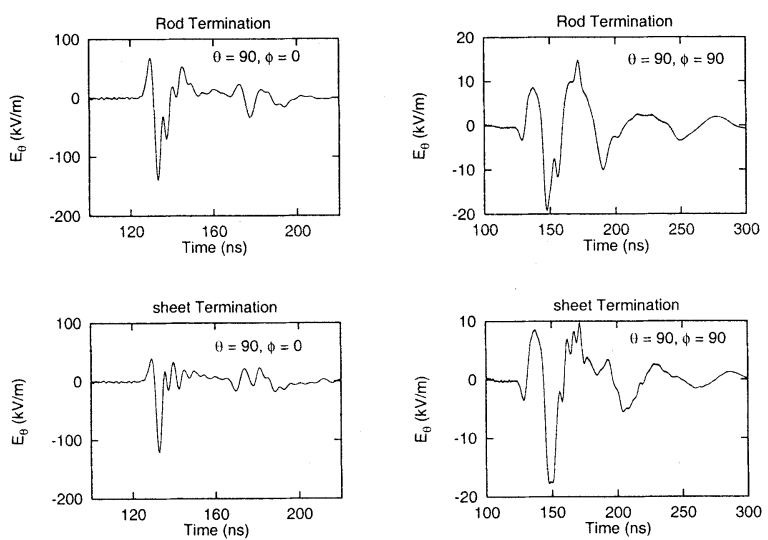

Fig. 9. Far-zone electric field, normalized to unit distance. Case with matched sheet and rod terminations and $\tau_{s}=19.7 \mathrm{~ns}$.

rod terminations, respectively. This means that $\sim 17 \%-22 \%$ of the initial stored energy in the capacitor, i.e., $35.7 \mathrm{~J}$, is lost by radiation and could cause damage to nearby equipment.

A matching termination is normally used to reduce reflection toward the pulsed-power source. While an unmatched termination would certainly increase this reflection, it would be interesting to explore if there are concomitant benefits in terms of reduced leakage. We find that a $30-\Omega$ sheet termination yields a total leakage of $\sim 6.9 \mathrm{~J}$, only marginally higher than that of a matching termination. This raises the possibility that a systematic study of the effect of termination resistance on leakage might yield a way to reduce leakage.

Fig. 9 shows the temporal evolution of the far-zone electric field $E_{\theta}$ produced by the simulator in the horizontal plane $(\theta=$ 90) as a function of angle $\phi$. These angles are defined in Fig. 1. At time $\mathrm{t} \sim 133 \mathrm{~ns}$, there is a strong negative peak at $\phi=0$. In both cases, the sidelobes of $E_{\theta}(\phi=90)$ are much weaker than in the boresight. There are significant differences between the two kinds of terminations - the rod termination yields higher peak values of $E_{\theta}$, and the far field also takes longer to decay to low levels.

The conclusion is that a matching sheet termination is beneficial from the point of view of reduced leakage, since it reduces the total radiated energy and the peak instantaneous power outflow. This also seems consistent with the idea of a sheet admittance proposed by Baum [10] for terminating high-frequency transmission lines. 


\section{PREPUlSE}

A prepulse, caused by the displacement current through the switch capacitance, can be significant when the charging time of the source capacitor is comparable to the switch closure time. Such a situation can arise in practical simulators, as in [2], where the charging time of the peaking section is $\sim 10 \mathrm{~ns}$, only $5-10$ times higher than the switch closure time.

Fig. 4, from an FDTD simulation, shows the existence of a prepulse in this system. An enlarged view is shown in Fig. 10, for the case of $\tau_{s}=19.7 \mathrm{~ns}$. The first peak is the main prepulse, which is followed by a reflection from the aperture. The time delay between the main (positive) and reflected (negative) peaks corresponds to the double transit time between the feed and aperture, a distance of $10.8 \mathrm{~m}$.

It is of interest to estimate the prepulse from simple considerations and compare it with the FDTD waveform. This is reported in the following.

The displacement current $\mathrm{I}_{D}$ is given by

$$
I_{D}(t)=C \frac{d V(t)}{d t}
$$

where $C$ is the effective switch capacitance and $V(t)$ is the charging voltage applied between the capacitor plates.

Since we do not know the actual $V(t)$ used in the experiments [2], we have arbitrarily chosen a half-Gaussian-like waveform given by

$$
V(t)= \begin{cases}V_{0} e^{-\alpha(t-\tau)^{2}}, & \text { if } 0<t \leq \tau \\ 0, & \text { if } t<0\end{cases}
$$

where $\alpha=(4 / \tau)^{2}$, and $\tau$ is the characteristic time of charging. This waveform means that the excitation is only applied until the Gaussian reaches its peak value. Accurate calculations using FDTD require that there be at least ten computational cells within a wavelength at the highest frequency of interest [6]. We, therefore, choose $\tau$ such that $|V(\omega)|$ falls by seven orders of magnitude as compared to its peak value at the highest frequency that can be handled by our FDTD mesh. We note that for a full Gaussian pulse, this decay by seven orders occurs at a frequency $f_{h} \simeq 6 / \tau$.

Given $I_{D}(t)$, the electric field prepulse $E_{p}(t)$ inside the structure can be estimated as follows. The input impedance $Z_{\text {in }}$ $(90 \Omega)$ of the structure remains constant over some frequency band because of its ultra-wideband nature. $E_{p}(t)$ developed at any point inside the structure can then be estimated from

$$
E_{p}(t)=\frac{I_{D}(t) Z_{i n}}{\operatorname{gap}}
$$

where "gap" is the separation between the plates of the TEM structure at the appropriate point.

Fig. 10 shows a comparison between the FDTD and synthetic prepulse waveforms. The switch capacitance $C$ has been calculated, using a separate FDTD calculation, as $2.25 \mathrm{pF}$. Here, we have accounted for the fact that the switch in our model actually consists of two resistive strips in series, which halves the capacitance. Three points must be noted. First, the synthetic waveform should only be compared with the forward-traveling FDTD prepulse and not with the reflected portion. Second, the synthetic

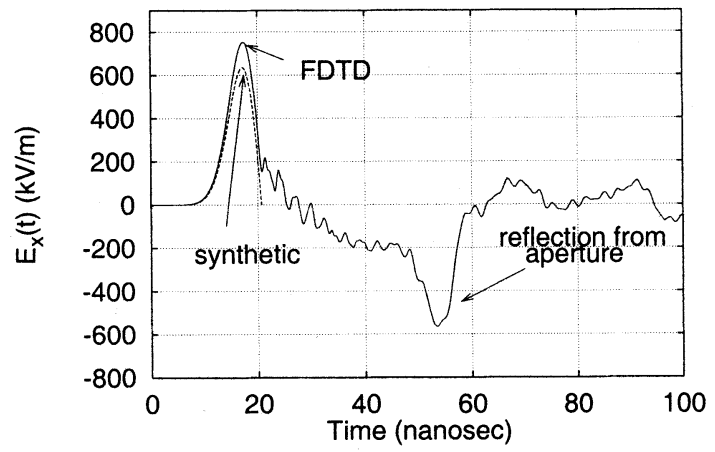

Fig. 10. Electric field prepulse.

prepulse falls to zero after the charging waveform reaches its peak. This is because, in our simulation, the charging waveform is not applied beyond this point. Third, we have taken a delay $\sim 1$ ns between $d V / d t$ of the charging waveform and the synthetic curve. This is because in the FDTD simulation, the electric field is being measured $\sim 0.3 \mathrm{~m}$ away from the closest point on the capacitor.

There is a fairly good agreement between the FDTD-computed main prepulse and the synthetic prepulse. This shows that the computation can be generalized to predict the prepulse from more complex switch geometries.

\section{EFFECT OF TEST OBJECT}

The test object is placed within the test volume formed by a parallel-plate extension of the tapered structure. The presence of the test volume and object is likely to affect two things. First, the total energy leakage from the system would depend upon the length of the parallel-plate section and also the size, shape, and material of the test object. It is essential to know the magnitude of this change from the point of view of EMI. Second, the test object would affect the electromagnetic field structure in its vicinity, especially in terms of the frequency spectrum. Since the simulator is supposed to subject the object to a particular kind of spectrum, any change in this distribution is of interest.

Test objects of practical interest often have complex features like internal cavities, small apertures, complicated geometries and multiple materials. Hence, the two issues mentioned cannot be studied by simpler methods-a 3-D FDTD calculation is necessary.

In the actual simulator, this extension is likely to be fairly large. Due to FDTD mesh constraints, we have limited ourselves to an extension $3 \mathrm{~m}$ long, having a width equal to that of the aperture in the tapered section, as shown in Fig. 1. This defines a test volume with the dimensions $3 \times 2.32 \times 1.49 \mathrm{~m}$.

In order to isolate the contribution of the test object, we have first performed the simulation with this extension, but without the object. We find a total energy leakage of $10.8 \mathrm{~J}$ for the case of sheet termination, which amounts to $\sim 30 \%$ of the initial stored energy in the capacitor. This marks an increase of $\sim 80 \%$ over the case without the parallel-plate extension.

Test objects come in a variety of geometries and materials. Our purpose here is only to estimate the kind of modification a test object can produce. Hence, we have examined a simple shape, viz., a perfectly conducting solid cube (see Fig. 1). The 

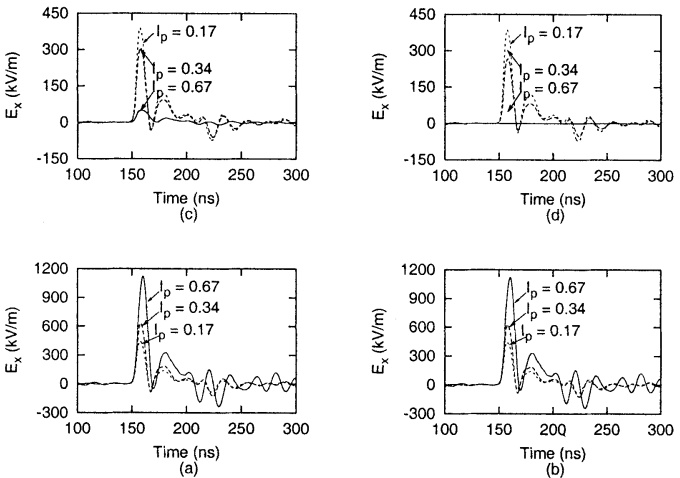

Fig. 11. Comparison of vertical component of electric field near different faces of the test object. Case with $\tau_{s}=19.7 \mathrm{~ns}$.

side of the cube $h_{t o}$ is allowed to take on three values, viz., 0.25 , 0.5 , and $1.0 \mathrm{~m}$, respectively. We define an "interaction parameter" $I_{p}\left(=h_{t o} / h_{\mathrm{pp}}\right)$, where $h_{\mathrm{pp}}(=1.49 \mathrm{~m})$ is the separation between the parallel plates.

Fig. 11(a) and (b) shows the vertical component of the electric field just outside the centers of the $x$-directed faces 1 and 2 . The electric field strength increases with increase in $I_{p}$ from 0.17 to 0.67 . The peak values scale approximately as the inverse of the gap between the object and the parallel-plates. Fig. 11(c) and (d) shows the vertical component of $\vec{E}$ just outside faces 3 and 4 , which are oriented normal to the $y$ direction. The field strength falls off with increase in $I_{p}$, indicating that $E_{x}$ gets effectively shorted. A similar trend in the electric field amplitudes has been found outside faces 5 and 6 .

For the values of $I_{p}$, there is no distortion in the vertical component of electric field inside the tapered section of the simulator. However, the $E_{x}(t)$ waveform near the test object is considerably distorted, with risetimes of 4.6, 4.76, and $6.1 \mathrm{~ns}$ for $I_{p}=0.17,0.34$, and 0.67 , respectively. This means that a larger test object will be subjected to somewhat lower frequencies, for identical simulator and pulser parameters. The simulator considered here can be used for testing objects with a maximum dimension of $\sim 0.5 \mathrm{~m}$, without significant change in the power spectrum.

We have also studied the effect of test object size on radiation leakage, for both rod and sheet terminations. The net energy outflow for test cubes of side $0.25,0.5$ and $1.0 \mathrm{~m}$, in the case of rod and sheet terminations, are 13.9, 13.8, $12.8 \mathrm{~J}$ and 10.8, 10.7, $9.9 \mathrm{~J}$, respectively. It appears that a larger test object decreases the net energy leakage for both kinds of terminations. However, the leakage is significantly higher in the case of rod termination.

An EMP simulator is meant to subject objects to fields similar to those that would be encountered when the pulse comes through free-space [3]. This necessarily requires the application of an E-field waveform at the TEM structure feed, similar in frequency content to the desired EMP waveform. However, even if the feed waveform is appropriate, scattering from various TEM cell components results in the object experiencing fields that are somewhat different from the desired form. It is important to quantify this difference.

We have compared the $E_{x}(t)$ waveforms, measured just above surface \#2 (defined in Fig. 1), for illumination in free space (FS) and inside the simulator (SI). The results are shown

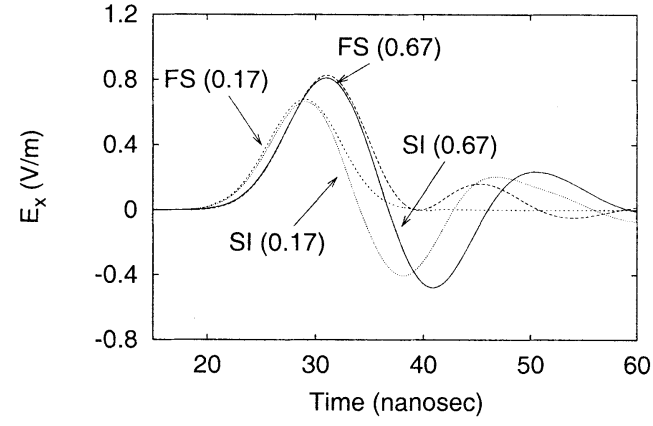

Fig. 12. Scattered $E_{x}(t)$ measured just above surface \#2 for illumination in free space (FS) and inside the simulator (SI). Curves are shown for $I P=0.17$ and 0.67 .

in Fig. 12 for $I_{p}=0.17$ and 0.67 . In all cases, the applied $E_{x}(t)$ is taken to be a Gaussian with unit amplitude, with $\tau=19.7 \mathrm{~ns}$. The SI results have been suitably scaled. As expected, we observe reasonable agreement between the FS and SI results up to the first peak. For both objects, subsequent scattering of fields off the TEM structure, especially the termination, produces negative peaks in the SI waveforms which are not present in the FS curves. We also observe that the SI response for the smaller object has a smaller 10\%-90\% risetime, indicating greater high-frequency content. This is consistent with our observations earlier in this section.

One point is noteworthy. The applied Gaussian, with $\tau=$ $19.7 \mathrm{~ns}$, has a $10 \%-90 \%$ risetime $\simeq 5.7 \mathrm{~ns}$. The $10 \%-90 \%$ risetimes observed above, for $I_{p}=0.17$ and 0.67 , are $\sim 5.9$ and 6.2 ns, respectively. This means that the smaller object essentially follows the risetime of the applied pulse, while the larger object exhibits a slower response. This is because the measured $E_{x}(t)$ has contributions from all points on the object, and these points are further away in the case of the large object. If the applied Gaussian had had a faster risetime, e.g., 2 ns, the finite transit-time through the smaller object would also have played some role, further enhancing the difference between risetimes for the two objects. This has already been observed in the case of the capacitor-driven simulator, described earlier in this Section.

\section{PHYSICAL INTERPRETATION}

FDTD simulations provide a detailed 3-D, time-dependent picture of the evolution of electromagnetic fields. We can, therefore, obtain a physical understanding of the results presented in Section VI. Since our focus in the last section was on radiation leakage, the required understanding can be obtained through an analysis of the Poynting flux distribution. For reasons of space, this study is limited to the case with $\tau_{s}=19.7 \mathrm{~ns}$ and a test object with side $1 \mathrm{~m}$.

Figs. 13 and 14 show 2-D vector plots of the Poynting flux for sheet and rod terminations, respectively. The vectors, comprising the $y$ and $z$ components $P_{y}$ and $P_{z}$, are represented in the $y z$ plane exactly between the top and bottom plates of the test volume shown in Fig. 1. In order to illustrate the role of the terminations, the vectors are shown over a region slightly bigger than the test volume.

Figs. 13 (a)-(d) and 14 (a)-(d) correspond to four different time snapshots, viz., $t=7.8 \tau$ to $8.4 \tau$ in steps of $0.2 \tau$, with 

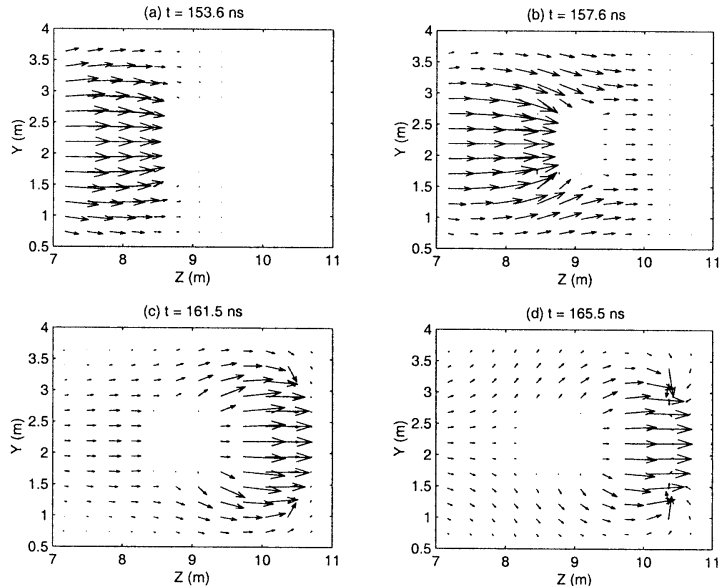

Fig. 13. Snapshots of Poynting flux distribution through the test volume, in the presence of a sheet termination and a test object of side $1 \mathrm{~m}$. Plots (a)-(d) correspond to $t=153.6,157.6,161.5$, and $165.5 \mathrm{~ns}$, respectively.
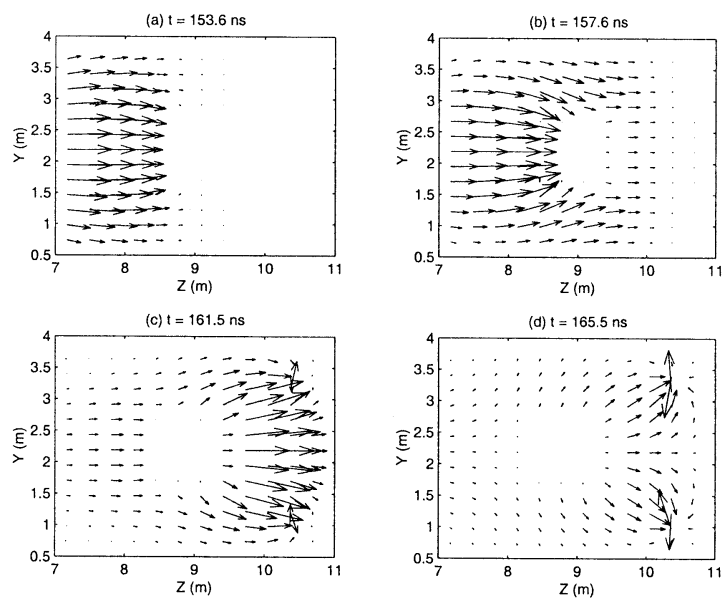

Fig. 14. Snapshots of Poynting flux distribution through the test volume, in the presence of a double-rod termination and a test object of side $1 \mathrm{~m}$. Plots (a)-(d) correspond to $t=153.6,157.6,161.5$, and $165.5 \mathrm{~ns}$, respectively.

$\tau=19.7 \mathrm{~ns}$. These time values have the following significance. The first is the time just before the main E-field peak reaches the front surface of the test object. The second is when the pulse just reaches the back surface of the object. Fig. 13(c) and 14(c) show the distribution shortly after the pulse crosses the termination, and Fig. 13(d) and 14(d) come after another 4 ns. Some points of interest are discussed in the following.

First, with both kinds of termination, the $P_{y}$ components are small on the $y$-directed boundaries, corresponding to weak sidelobes, consistent with the observation made from Fig. 9.

Second, Figs. 13(a)-(b) and 14(a)-(b) are similar in both figures. This is to be expected, considering that the main positive peak has not yet reached the termination. However, a portion of the pulse preceding the main peak would have reached the termination, and this accounts for minor differences of detail between Figs. 13(b) and 14(b).

Third, in both subplots (b), we note that the incoming flux lines tend to bend toward the test object. This can be understood as follows. The applied $E_{x}$ would induce transient $x$-directed currents on both $y$-directed faces of the object. Idealizing these as sheet currents in the $x$ direction, we would get equal and opposite magnetic field components $H_{z}$ outside the two faces. It is easily seen that the resulting $P_{y}$ would point toward the object on both faces, giving rise to the observed "inward bending" of the power flow.

Another interesting difference can be seen between the Figs. 13(c) and 14(c), corresponding to a time shortly after the main pulse crosses the termination. Near the sheet termination [Fig. 13(c)], the Poynting flux has primarily a zvcomponent, while a rod termination results in substantial $y$-directed divergence of the power "flow." This can be interpreted in terms of the magnetic field produced by the $x$-directed current through the terminations. A sheet termination would produce primarily $H_{y}$ inside the bounded volume. This, in combination with $E_{x}$, would yield primarily $P_{z}$. A rod, on the other hand, would produce an azimuthal magnetic field around itself, containing both $H_{y}$ and $H_{z}$. Inside the bounded volume, the resulting $H_{z}$ would have equal and opposite values at the two limiting values of $y$, giving rise to the observed divergence.

Finally, we see from Fig. 13(c) and (d) that there is a significant reduction in the magnitude of the Poynting flux just outside the sheet termination, as compared to its value inside. With a rod termination, the decrease is not so marked. This explains the observed reduction in total energy leakage when a sheet termination is used.

\section{MAJOR LIMITATIONS OF THE STUDY}

We have modeled an idealized form of the EMP simulator described in [2]. The peaking section in [2] consists of several water capacitors. We have taken a single air-gap, parallel plate capacitor, but with similar capacitance. The difference in dielectric material and geometry means that transmission-line effects within the capacitor will not be handled realistically. However, this should affect only very short time-scale phenomena.

No details of the switch are available from [2]. Hence, we have assumed a simple model. This means that the computed prepulse and the early-time part of the field in the horn may not match experimental values.

The simulator in [2] has a large test volume beyond the pressurized section. Inclusion of the complete test volume would require a very large increase in the computational domain, while the mesh size would be restricted to small values due to the need to handle high frequencies. Computer hardware limitations do not presently allow us to handle the full problem. Hence, we have assumed only a 3-m-long test section. Even with this restriction, we are unable to use a mesh size finer than $1.85 \mathrm{~cm}$, which permits a maximum frequency of $1.6 \mathrm{GHz}$. The fastest $10 \%-90 \%$ risetime obtained in our simulations is $2.2 \mathrm{~ns}$, which can be handled by this mesh. However, the fastest experimental risetime (20\%-90\%) reported by [2] is $1 \mathrm{~ns}$. There is a need to stretch this limit by using a finer mesh.

\section{CONCLUSION AND Future Work}

We have performed self-consistent, 3-D, time-domain calculations for a bounded-wave EMP simulator, consisting of a constant-impedance TEM structure driven by a charged capacitor. To our knowledge, this is the first time such a study has been done. The simulations yields the detailed 3-D evolution of 
electromagnetic fields within the structure and in its immediate vicinity. As expected, the pulse travels along the length of the simulator with a nearly constant pulsewidth and risetime. The peak amplitude falls in inverse relation to the height of the TEM structure and is constant in the parallel-plate test region of the simulator.

For the parameters of [2], we expect a significant prepulse. This is indeed seen in the simulations. The prepulse waveform can be explained fairly well quantitatively, using simple estimates of capacitive coupling across the switch and the known charging waveform across the capacitor.

Placement of a test object within the test section significantly modifies the electric field "seen" by the object. The vertical field $E_{x}$ experienced by the $x$-directed faces of the object increases with object size, while $E_{x}$ outside the $y$-and $z$-directed faces shows the reverse trend. $E_{x}(t)$ on the surface of the object shows progressively larger risetimes as the object size is increased, i.e., larger objects will be subjected to somewhat lower frequencies. However, this increase in risetime becomes significant only beyond some critical object size. On the positive side, larger test objects appear to marginally reduce radiation leakage from the system. We have also provided a physical interpretation for some of these results, in terms of power flow through the volume. The E-field waveform experienced by a small test object is reasonably close to that for free-space illumination, but the mismatch increases with object size.

As expected, shorter closing times for the switch yield shorter risetimes for the electric field at the feed point of the simulator. However, as closure times become smaller, the risetime does not decrease proportionally, possibly due to inductive effects from the connections and switch.

The use of a resistive sheet as a matching termination significantly reduces radiation leakage as compared to two parallel resistive rods. We next plan a systematic study of the effect of termination resistance, and also the geometry of the termination, on the leakage.

Computer hardware limitations have restricted this study to risetimes that are a factor of two longer than those produced experimentally in such systems [2]. It is planned to extend the study for frequencies up to few gigahertz, by using a finer computational mesh and running the code on a parallel computer. Future work will also make use of submeshing and variable meshing, which will allow a fine mesh near the capacitor, switch and feed point and a progressively larger mesh as we move toward the aperture-this should help reduce the computational cost. An effort is also planned to incorporate more realistic models of the closing switch.

\section{ACKNOWLEDGMENT}

The authors are grateful to the referees, whose suggestions helped to materially improve this paper. They would like to acknowledge valuable discussions with Prof. P. I. John and Dr. H. Ramachandran.

\section{REFERENCES}

[1] H. M. Shen, R. W. P. King, and T. T. Wu, "The exciting mechanism of the parallel-plate EMP simulator," IEEE Trans. Electromagn. Compat., vol. EMC-29, pp. 32-39, Feb. 1987.
[2] H. Schilling, J. Schluter, M. Peters, K. Neilsen, J. T. Naff, and H. G. Hammon, "High voltage generator with fast risetime for EMP simulation," in Proc. 10th IEEE Int. Pulsed Power Conf., Albuquerque, NM, 1995, pp. 1359-1364.

[3] C. E. Baum, "EMP simulators for various types of nuclear EMP environments: An interim categorization," IEEE Trans. Antennas Propagat., vol. AP-26, pp. 35-53, Jan. 1978

[4] C. Bardet, O. Dafif, and B. Jecko, "Time-domain analysis of a large EMP simulator," IEEE Trans. Electromagn. Compat., vol. EMC-29, pp 40-48, Feb. 1987.

[5] K. M. S. Hoo, "Numerical analysis of a transmission line EMP simulator," IEEE Trans. Electromagn. Compat., vol. EMC-17, pp. 85-92, May 1975.

[6] K. S. Kunz and R. J. Luebbers, The Finite-Difference Time-Domain Method for Electromagnetics. Boca Raton, FL: CRC, 1993.

[7] K. L. Shlager, G. S. Smith, and J. G. Maloney, "Accurate analysis of TEM horn antenna for pulse radiation," IEEE Trans. Microwave Theory Tech., vol. 38, pp. 414-423, Aug. 1996.

[8] T. S. Utton, "Ultra-wideband TEM horns, transient arrays and exponential curves: An FDTD look," Master's thesis, Air Force Inst. Technol., Wright-Patterson AFB, Dayton, OH, 1999.

[9] A. R. Dick, S. J. MacGregor, A. J. McPhee, M. T. Buttram, R. C. Pate, P. E. Patterson, L. F. Rinehart, and K. R. Prestwich, "An investigation into high speed gas breakdown," in Proc. 23rd Int. Power Modulator Symp. 1998, pp. 202-205.

[10] C. E. Baum, "Admittance sheets for terminating high-frequency transmission lines," Sensor Simulat. Notes, Air Force Inst. Technol., CA Inst. Technol., vol. SSN 53, Apr. 18, 1968.

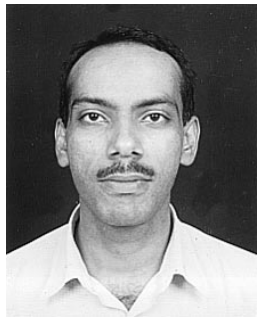

Shahid Ahmed received the B.Sc. and M.Sc. degrees in physics from Ranchi University, Ranchi, India, in 1993 and 1995, respectively. His specialization during the M.Sc. studies was electronics and microwaves. He is currently working toward the Ph.D. degree in the computational study of electromagnetic pulse (EMP) simulators at the Institute for Plasma Research, Gandhinagar, India.

His research interest is the computer simulation of pulsed-power systems including electromagnetics, plasma physics, and its application to fast switches.

Mr. Ahmed has been a student member of the IEEE Antennas and Propagation Society since 2002 .

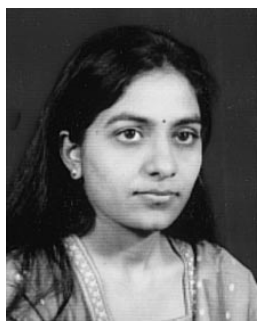

Deepti Sharma received the B.Sc. degree in physics from Vikram University, Ujjain, India, in 1997, and the M.Sc. degree in nuclear physics from Devi Ahilya University, Indore, India, in 1999.

Currently, she is involved in the computer simulation of electromagnetics with the Pulsed Power Group of the Institute for Plasma Research, Gandhinagar, India. Her research interests include the computational study of electromagnetics and fluid dynamics.

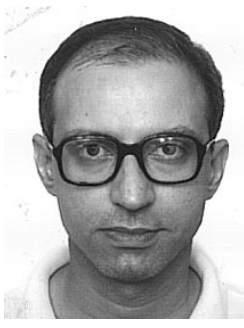

Shashank Chaturvedi received the B.Tech. degree in chemical engineering from the Indian Institute of Technology, Delhi, India, in 1985, and the Ph.D. degree in chemical engineering from Princeton University, Princeton, NJ, in 1989. His research involved the computational study of a novel fusion reactor configuration.

He has since been with the Institute for Plasma Research, Gandhinagar, India. For some years, he worked on the computational modeling of tokamaks. Over the past eight years, he has been involved in the modeling of pulsed-power systems, including radiation-hydrodynamics and MHD simulations, as well as shockwave studies. 